\title{
La precarización del trabajo desde una perspectiva sociocultural en un contexto fronterizo
}

\author{
Marlene Solís*
}

Resumen: en este artículo se analiza la conformación del mercado de trabajo de la industria del vestido en Tijuana; se identifican los factores estructurales generadores de precariedad y, según las vivencias respecto a ella, se reconstruyen perfiles distintos de los y las trabajadoras. El objetivo de esta propuesta analítica es mostrar la manera en que se entrelazan las escalas micro, meso y macrosocial reproduciendo o produciendo las condiciones de precariedad que prevalecen en los empleos de la industria de la confección en Tijuana. Para interpretar la experiencia de la precariedad, se aborda su dimensión sociocultural, al darle un papel central a los significados que le otorgan las personas al trabajo.

Palabras clave: precarización laboral, migración y género, industria de la confección, maquiladoras, frontera norte.

Abstract: this article analyzes the configuration of the garment industry labor market in Tijuana. The structural factors which generate job insecurity are identified, and, depending on personal experiences, different profiles are reconstructed

\footnotetext{
* El Colegio de la Frontera Norte (COLEF). Km 18.5 carretera Escénica Tijuana-Ensenada, San Antonio del Mar, C. P. 22560, Tijuana, Baja California, México. Teléfono: (664) 6316300 , extensión 3206. Correo electrónico: msolis@colef.mx
} 
for male and female workers. The analysis proposed seeks to show the way in which the micro-, mezzo- and macrosocial scales intertwine, reproducing or producing the conditions of job insecurity that prevail in this industry. The experience of job insecurity is seen from a sociocultural perspective, giving a central role to the personal meanings of work.

Key words: job insecurity, migration and gender, garment industry, maquiladoras, northern border.

\section{Introducción}

El objetivo de este artículo es analizar, desde una perspectiva sociocultural, los procesos que han dado lugar a la precarización del mercado de trabajo de la industria de la confección de prendas de vestir en Tijuana. ${ }^{1}$ Aquí, la precarización laboral se concibe como la tendencia hacia una mayor inestabilidad del empleo, el acceso cada vez más restringido a la seguridad social y la persistencia de salarios bajos. Estas condiciones, a su vez, implican el debilitamiento del empleo como medio para garantizar el ejercicio de los derechos sociales: trabajo digno, educación, vivienda y salud. Por tanto, genera incertidumbre respecto al futuro y conlleva el riesgo de perder un estatus social o de caer en una situación de pobreza y exclusión (Castel 1997).

Como antecedente al concepto de precariedad se identifican las ideas respecto a la noción de heterogeneidad de la estructura del empleo, que surge como parte de la teoría de la dependencia. ${ }^{2} \mathrm{La}$ heterogeneidad estructural se refiere a la existencia de diversas for-

\footnotetext{
${ }^{1}$ Los resultados presentados en este artículo forman parte del proyecto de investigación "Heterogeneidad ocupacional, precariedad laboral y diferencias de género", financiado por el Consejo Nacional de Ciencia y Tecnología, Ciencia Básica 2008-2011; participaron investigadores del COlEF, El Colegio de México (COLMex) y de la Universidad Autónoma MetropolitanaIztapalapa.

${ }^{2}$ Propuesta a finales de los años sesenta por autores ligados a la Comisión Económica para América Latina (CEPAL).
} 
mas o modos de producción, como rasgo característico de los países en desarrollo, lo cual sería entonces lo que distingue al capitalismo de la periferia, del que se conocía como el "clásico", de las naciones desarrolladas. ${ }^{3}$ Dicho concepto (véase Pacheco 2004) hace referencia a dos grupos de trabajadores distintos que dan lugar a nociones como subempleo, marginalidad e informalidad, para entender formas de empleo no asalariadas, por cuenta propia y, por lo general, precarias, consideradas como actividades productivas no incorporadas o fuera de la lógica de producción capitalista.

De esta manera, la estructura heterogénea del empleo alude a la persistencia de una porción significativa de trabajadores no asalariados, tendencia que parecía contraria a los procesos de salarización creciente que se había dado en los países desarrollados y que el modelo de desarrollo, basado en la sustitución de importaciones, pretendía alcanzar junto con las políticas del Estado benefactor. Sin embargo, en México el empleo formal o estándar nunca fue el típico en términos sociológicos ni estadísticos (Rojas y Salas 2008).

El debate en cuanto al concepto de trabajo precario se inició en Europa a principios de la década de 1970, mientras que en México el de precariedad laboral adquirió importancia en años recientes. Esta aparente falta de interés sobre el tema se debió a la heterogeneidad del mercado de trabajo en el país y a la asociación del empleo no asalariado con las naciones mencionadas, con el subempleo, la marginalidad, la informalidad y con el trabajo precario.

Sin embargo, con la crisis de los años ochenta, el aumento de las actividades no asalariadas en el país y el estancamiento del empleo asalariado y la flexibilización del trabajo se empezaron a evidenciar cambios en la asociación directa entre trabajo no asalariado con precariedad laboral, ${ }^{4}$ que cuando se extiende a las ocupaciones asa-

\footnotetext{
${ }^{3} \mathrm{Al}$ analizar los efectos de la globalización en el empleo en México, Guillén (2005) señala que la heterogeneidad estructural en vez de atenuarse, con el nuevo modelo de desarrollo, se ha ampliado y ha vuelto complejas las relaciones entre el sector moderno y el atrasado.

${ }^{4}$ Uno de los estudios que presenta elementos para modificar la manera de entender el vínculo conceptual y empírico entre trabajo no asalariado y precariedad es el de Pacheco (2004), quien encuentra en su análisis sobre la heterogeneidad y las condiciones de trabajo en la Ciudad de México, durante el periodo 1989-1998, que para los hombres el trabajo no asalariado en algunas ramas de los servicios constituía una mejor opción de remuneración frente al asalariado. En cambio, esto no ocurre para las mujeres, para quienes el trabajo no asalariado sigue representando salarios menores.
} 
lariadas hace necesario profundizar en el conocimiento acerca de los factores específicos que dan lugar a la precarización y su relación con la heterogeneidad estructural. ${ }^{5}$

En las últimas décadas -como plantean Rojas y Salas (2008)recibir bajos ingresos se ha convertido en una característica generalizada en la fuerza de trabajo mexicana, en parte debido a que la competitividad del mercado se ha logrado mediante la contención salarial, con la apertura de la economía. Por lo tanto, los bajos salarios están entre los principales rasgos de la precariedad laboral en el país. En esta tendencia, el empleo en las maquiladoras ha sido punta de lanza, pues la precariedad de los empleos ha persistido desde la instalación de las primeras plantas hasta hoy cuando, a la luz de la crisis económica mundial del siglo xxi, las condiciones laborales continúan deteriorándose.

Así, en la presente investigación empírica de la industria de la confección en la frontera norte de México, interesa describir cómo se ha conformado este mercado de trabajo, considerando tanto la heterogeneidad estructural en la industria de la confección de Tijuana, las prácticas de flexibilización de las empresas y, en un segundo momento, la precariedad vivida. Esta propuesta responde a la idea de que los actores negocian los condicionantes sociales, en este caso la precariedad, y generan procesos de reproducción y producción de nuevas alternativas que obstaculizan o posibilitan el mejoramiento y dignificación del empleo.

De este modo, la precarización del trabajo expresa una reconfiguración de las desigualdades y de las relaciones sociales, es decir, que a las diferencias "clásicas" entre categorías socio-profesionales se superponen otras sociales dentro de cada una, lo que tiende a aumentar la precariedad (Avenel y Thibault 2006). Por ello, la investigación sobre la situación actual de la precariedad laboral, a partir de estadísticas y del análisis del mercado de trabajo a escala nacional, permite capturar de manera parcial lo que ocurre, y es necesario adoptar una aproximación más dinámica de las desigualdades laborales que considere los cambios estructurales, así como en las empresas, las

\footnotetext{
${ }^{5}$ Para 2006, García (2009) caracterizó al mercado de trabajo en México, y distinguió los elementos siguientes: 60.8 por ciento de la población económicamente activa (PEA) no percibía prestación alguna, 73.6 no tenía contrato permanente y 90.6 de los trabajadores no estaban sindicalizados.
} 
trayectorias individuales y la diversidad de las posiciones sociales de las y los trabajadores, que se tornan más móviles e inestables.

En la propuesta analítica se considera también la importancia del individualismo contemporáneo y el debilitamiento del Estado benefactor, como rasgos del nuevo modelo económico y social, que explican la precarización del trabajo al generar un contexto de incertidumbre, riesgo, vulnerabilidad y la multiplicación de las discontinuidades en los cursos de vida. La inestabilidad y movilidad de la sociedad tienen relación tanto con las mutaciones macroestructurales del capitalismo, como con la transformación de las relaciones vitales y la manera de formarse una identidad. Por lo que es necesario incorporar al análisis la experiencia vivida de las personas, con la finalidad de considerar los recursos diferenciados y la capacidad de negociación de los sujetos frente a los condicionantes sociales.

Por otra parte, se propone el análisis de un mercado local de trabajo, que considere que los contextos regionales y locales juegan un papel fundamental en la definición de la tendencia hacia la precariedad laboral, que además es útil para distinguir las particularidades e identificar distintos mecanismos asociados al territorio, que intensifican o mitigan esta tendencia. La relevancia de considerar la frontera norte, como caso de estudio, se debe al papel histórico de esta región en la apertura de la economía nacional y los procesos de relocalización industrial en el pasado reciente. Asimismo, el mercado de trabajo de la industria de la confección representa uno de los sectores productivos más golpeados por las crisis mundiales en la primera década de este siglo, y las estrategias empresariales para hacer frente a tales circunstancias han incluido formas distintas de flexibilidad laboral. Por lo que este mercado constituye un objeto de estudio pertinente para el propósito general de esta investigación, que es aportar elementos nuevos para entender la tendencia hacia la precariedad, como expresión de un conjunto de procesos que actúan a escalas y ámbitos de vida distintos.

La metodología utilizada en la investigación empírica consistió en la revisión de fuentes secundarias de información, ${ }^{6}$ para dar cuenta

\footnotetext{
${ }^{6}$ Los censos de población y vivienda, los censos económicos del Instituto Nacional de Estadística y Geografía (INEGI), y los informes de la Cámara Nacional de la Industria del Vestido (CNIv) y otros estudios sobre del sector de la fabricación de prendas de vestir.
} 
de la dinámica de la industria de la confección en México y en Tijuana en particular; también se aplicó un cuestionario a 27 personas, y a partir de las trayectorias laborales ${ }^{7}$ reconstruidas se realizaron -en un tercer momento- nueve entrevistas semiestructuradas a un grupo de hombres y mujeres empleados en este ramo. ${ }^{8}$

El primer apartado consiste en la argumentación sobre los elementos teóricos para el análisis de la precariedad, desde una perspectiva sociocultural. A partir de estas ideas, en el segundo se describen los procesos específicos que dan lugar a la precariedad laboral en la industria de la confección a escala nacional y en Tijuana. En el tercero se presentan las raíces de la diversidad laboral en este mercado de trabajo, ya que es necesario considerar las diferentes orientaciones hacia él para entender la precariedad vivida, que se analiza en la última parte considerando casos paradigmáticos de acuerdo con la edad, el género y la condición migratoria.

Para concluir se presenta una reflexión sobre los hallazgos más importantes acerca de los procesos de precarización en un mercado de trabajo local y las distintas formas individuales de enfrentar estos procesos, las cuales permiten la continuidad de las condiciones que llevan a la precariedad.

\section{La precarización laboral desde una perspectiva sociocultural}

Con el fin de definir una perspectiva sociocultural de la precarización laboral, es necesario considerar que la tendencia hacia el deterioro de la calidad de los empleos es el resultado de la conjugación de procesos económicos, sociales y culturales; los cuales operan en escalas macro, meso y microsocial, y que pueden tener un carácter

\footnotetext{
${ }^{7}$ Aquí no se incluyó el análisis de las trayectorias laborales, que puede consultarse en López et al. (2011 y 2012) y Solís y Castañeda (2013).

${ }^{8}$ El cuestionario se aplicó a 17 mujeres y 10 hombres, en el caso de las entrevistas fueron 6 mujeres y 3 hombres. Los criterios de selección de los informantes se establecieron con la intención de capturar la diversidad de la fuerza de trabajo, por tanto, se incluyó a jóvenes, adultos, hombres y mujeres con distinto estado civil. Los cuestionarios se levantaron a la salida de las fábricas y las entrevistas se concertaron a través de la técnica bola de nieve.
} 
estructural debido a su imbricación y peso en las relaciones sociales en un tiempo y contexto específicos.

En segundo lugar, se parte de los planteamientos de la sociología del conocimiento, referentes a la construcción social de la realidad, para entender la configuración de los mercados de trabajo. Por lo tanto, éstos se conciben como el resultado de la interacción cotidiana de agentes que demandan u ofrecen fuerza de trabajo, con el fin de llevar a cabo actividades productivas. En esta interacción se reproducen y producen tanto los aspectos materiales como simbólicos, que permiten realizar determinada actividad productiva. Como señala Reygadas (2002), a toda producción material le corresponde una simbólica, ya que los actores le otorgan sentido a sus acciones mediante un conjunto de sistemas de significación.

Esta idea supone que la precariedad que caracteriza a ciertos empleos tiene una dimensión objetiva y una subjetiva, es decir, que si bien es posible clasificarlos como precarios a partir de distintos indicadores, la percepción de la precariedad puede distanciarse de esta situación objetiva, ya que se encuentra mediada por las normas, valores y creencias de los individuos, interiorizados a lo largo de su experiencia vivida. Entonces, se entiende que la percepción de la precariedad ${ }^{9}$ es una construcción cultural que depende de las formas de identificación en el trabajo, así como de la trayectoria laboral y de vida de los individuos.

Así, la inserción laboral en un mercado de trabajo -clasificado por los especialistas como precario- adquiere significados diversos, pues en el contexto de la trayectoria individual de trabajo puede interpretarse como una mejora en relación con la experiencia laboral o vital previa. Entonces, en el plano microsocial, y desde la experiencia, la precariedad es relativa y situacional, ya que se inscribe dentro del curso de vida y trayectoria laboral de las personas.

Por otro lado, los actores tienen distintos recursos sociales y culturales que en un momento dado despliegan para enfrentar condiciones adversas, como puede ser la mayor inestabilidad de los em-

\footnotetext{
${ }^{9}$ De acuerdo con Avenel y Thibault (2006), la precariedad laboral designa una situación objetiva e implica un sentimiento de inseguridad social, de temor a caer en pobreza, de modo que no sólo se trata de realidades empíricas de carencia y bajos ingresos, sino la vivencia de inseguridad, de riesgo permanente.
} 


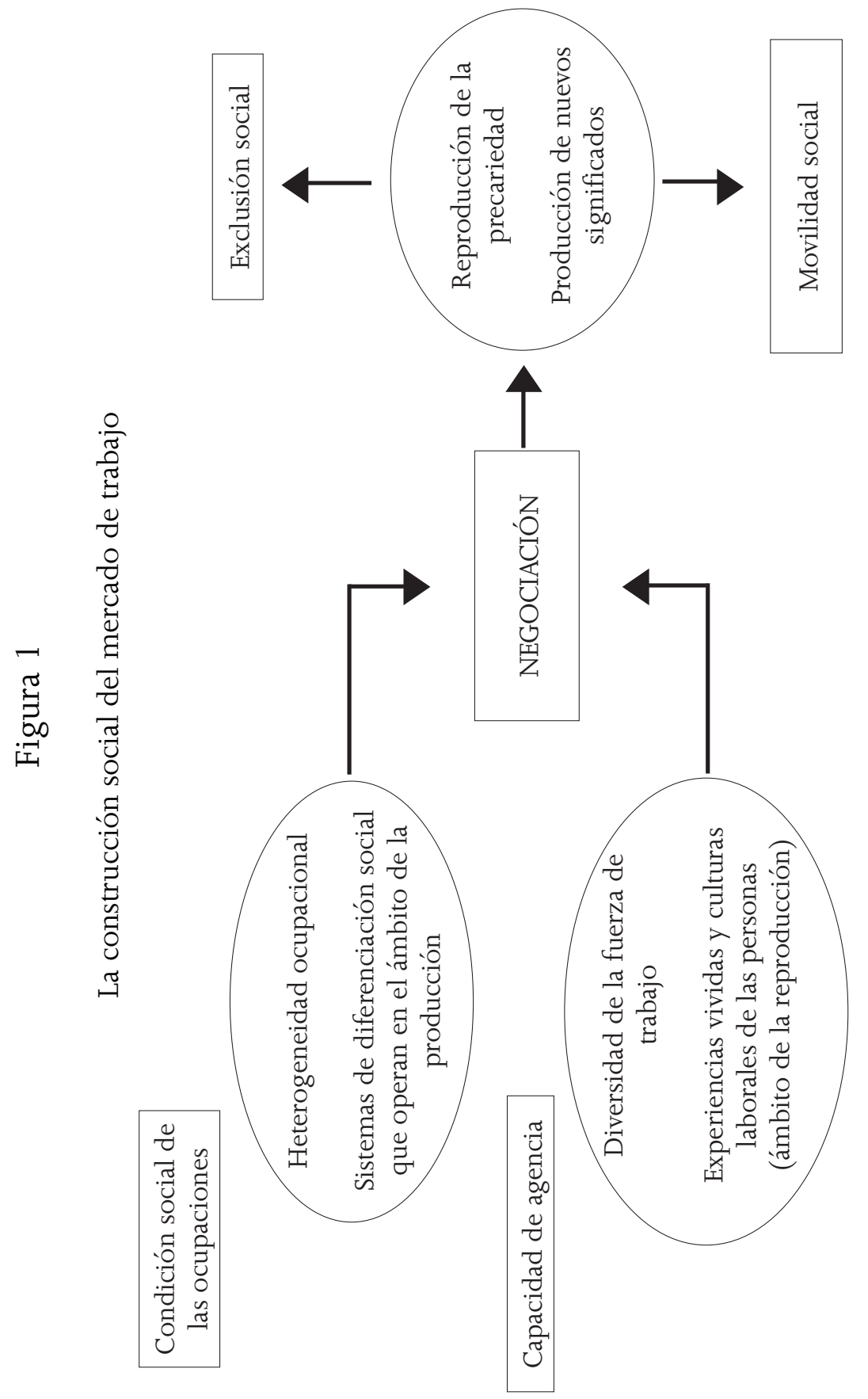

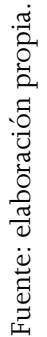


pleos, la dificultad para acceder a la seguridad social, la persistencia de salarios bajos y la imposibilidad de mejorar las condiciones de vida. La capacidad de respuesta, es decir, la agencia de las personas da lugar a un proceso de negociación y elaboración de distintos contenidos a la cotidianeidad, en un proceso dinámico de acciones que les permite sobrellevar, trasgredir, modificar o replegarse frente a la condición social de su ocupación y a la precarización del trabajo. En la figura 1 se muestran los planteamientos anteriores.

El interés en este estudio es analizar las condiciones objetivas y subjetivas de la precariedad laboral, por lo que se dará cuenta de cómo se expresan los procesos de precarización laboral identificados en otras investigaciones en un mercado de trabajo local, tales como la heterogeneidad estructural, la persistencia de bajos salarios y la condición social de jóvenes y mujeres (Mora y Oliveira 2010; Salas y Rojas 2008). Aquí es fundamental exponer el papel que juegan las prácticas de flexibilidad laboral, que implementan las empresas maquiladoras en una escala meso, por lo que dicha flexibilidad es una variable que atraviesa el análisis, ya que son estas prácticas las que dan lugar tanto a la reproducción de la heterogeneidad estructural como de las condiciones de precariedad laboral. Paralelamente, la diversidad constituye una característica esencial del mercado de trabajo, lo cual en principio se relaciona con los procesos de feminización de éste, que ocurrieron con la relocalización industrial en los países menos desarrollados; pero también, como se verá más adelante, en la región fronteriza de Tijuana la inmigración constituye un elemento fundamental de dicha diversidad.

\section{Los factores de precarización laboral en la industria de la confección}

En los últimos años, la industria textil y del vestido se ha transformado considerablemente en México debido a su inserción en la economía global, a través de la creciente subcontratación internacional de empresas. El dinamismo que generó la apertura al mercado externo en este sector, sobre todo durante los años noventa, implicó un as- 
censo en su actividad productiva, por lo que a finales de esa década llegó a ser la cuarta en importancia por su producción manufacturera y la primera en la generación de empleos. Además, se convirtió en una fuente de divisas de primer orden, pues se ubicó como la segunda rama por su volumen de exportaciones (García 2004).

Sin embargo, este sector ha tenido un desempeño desfavorable y enfrenta una perspectiva adversa, a consecuencia de los problemas presentes en su trayectoria; ${ }^{10}$ la creciente competencia internacional a la que se enfrenta (China) y la crisis económica. Así, el comportamiento del producto interno bruto de la cadena de valor textilvestido-cuero tuvo una fuerte caída, del año 2000 a 2003, logró estabilizarse hasta 2008, cuando empezó de nuevo a contraerse de tal modo que en 2009 tuvo la producción más baja en su historia reciente, pues se ubicó por debajo de la de 1995 (CNIv 2011).

En lo que respecta a los empleos en la fabricación de prendas de vestir, según datos de García (2004), se registró una pérdida de cerca de 185 mil del año 2000 a 2003. De 2008 a 2009 fue el lapso más crítico del sector, cuando se perdieron 24992 plazas laborales, lo que representó 8 por ciento del empleo total, y sólo en este año cerraron 667 empresas (CNIV 2011). En 2009 había 9312 industrias del ramo en todo el país, de las cuales han predominado las micro, que en esa fecha representaban 74 por ciento, 17 las pequeñas, 7 las medianas y 2 las grandes. ${ }^{11}$

Las prácticas para flexibilizar la producción han implicado cambios en su organización y nuevas formas de gestión de la fuerza de trabajo. Las líneas de producción en masa se han transformado en las que fabrican pequeños lotes diferenciados y de rápida programación, lo que ha dado lugar a la mayor segmentación de las tareas y a la creciente importancia del ensamble de piezas, como la actividad preponderante en las empresas deslocalizadas en países como México. Esta restructuración productiva resultó a su vez en la con-

\footnotetext{
${ }^{10}$ Según García (2004), los problemas estructurales son: el rezago tecnológico, la falta de diseño propio, escasez de personal capacitado, la poca integración de los eslabones de la cadena productiva textil, además de la caída del mercado interno, la falta de financiamiento a las empresas, la creciente participación en el mercado de la industria ilegal y los costos derivados de la inseguridad.

${ }^{11}$ Las microempresas tienen hasta 10 empleados, las pequeñas entre 10 y 50 , las medianas entre 50 y 250 y las grandes más de 250 .
} 
formación de cadenas globales. ${ }^{12}$ En un principio se ubicaron en la región pionera o de tradición maquiladora en la frontera norte, cuya importancia se extiende desde mediados de los años sesenta hasta finales de los ochenta, cuando fue notoria la expansión hacia otras zonas, que coincidió además con cambios en la política industrial mexicana, que generaron las condiciones necesarias para la instalación de este tipo de planta en otras latitudes del país. A partir de la movilidad de estas empresas se definió un segundo eje de ensamble, y esta reorganización territorial ocurrió principalmente en las ciudades que resintieron la caída de su actividad económica dando lugar a un excedente de fuerza de trabajo (De la O 2006).

Durante los años noventa, la industria maquiladora transitó por una época de auge, lo cual se debió en buena medida a las facilidades que brindó el Tratado de Libre Comercio, firmado en 1994 con Estados Unidos y Canadá. En este ciclo, en la región tradicional se gestó una especialización productiva, pues hubo mayor crecimiento del sector textil y del vestido en las regiones de expansión y emergentes, mientras que la electrónica y de autopartes adquirieron mayor importancia relativa, sobre todo, en urbes como Ciudad Juárez (autopartes) y Tijuana (electrónica). Además, en dicha década se conformó un movimiento más intenso de estas empresas hacia el centro-occidente y sur del país. Se observó el traslado de la industria de la confección hacia ciudades no fronterizas, y con ello se conformó la región emergente de la maquiladora (norte, occidente, centro y península de Yucatán).

En los últimos años, la recesión económica de Estados Unidos y México ha impactado con particular intensidad en el empleo y el poder adquisitivo del salario, por lo que en Tijuana se han presentado altas tasas de desempleo ${ }^{13}$ y empobrecimiento de la po-

12 El desarrollo productivo de esta industria, de acuerdo con la teoría de las cadenas de valor internacional (Bair y Gereffi 2001), se logra con la expansión de las maquiladoras, que de la confección transite al paquete completo, es decir, que se dé una mayor participación de los proveedores de fibras y textiles y se incorporen otros procesos, que permiten aumentar la generación de valor, como ocurrió con algunas fábricas de pantalones de mezclilla en Torreón. Según el INEGi (2009), 59 por ciento de las unidades económicas en la fabricación de prendas de vestir operan como productores, 27 sólo maquila las prendas, 12 es mixto y 1 es auxiliar.

13 Según los datos del INEGi (2009 y 2011), las tasas de desocupación en Tijuana, en el primer trimestre del año, se han ido incrementado: en 2008 fue 3.22, en 2009 de 7.42, en 2010 de 6.1 y el dato preliminar para 2011 fue de 7.49 por ciento. 
blación. En esta ciudad, el mercado de trabajo de la industria de la confección, si bien representa un porcentaje menor comparado con la electrónica, es un referente importante pues se trata de una de las actividades productivas más heterogéneas y donde la flexibilidad laboral se presenta con mayor amplitud.

El modelo de producción de pequeñas y medianas empresas en Tijuana

La industria de la confección se caracteriza por su heterogeneidad estructural, lo cual se refleja en las diferencias según el tamaño de las empresas, y eso a su vez implica sistemas productivos, relaciones laborales y condiciones de trabajo distintos. La característica común de las empresas es que se basan en el ensamble, a pesar de que a mediados de la década de 1990 empezaron a tener cierta importancia los procesos productivos más complejos; algunas de las que tienen esquemas productivos llamados de paquete completo se instalan, sobre todo, en la región lagunera (Guadarrama et al. 2010).

En Tijuana han predominado los establecimientos pequeños y medianos, según los datos del INEGI (2011); en 2010 había 197 registrados en el ramo de fabricación de prendas de vestir, entre los cuales sólo dos empleaban a más de 250 personas (grandes), 12 eran medianos, 13 pequeños y 170 micro, entre sastrerías, modistas, bordados y numerosos talleres de costura a domicilio. De acuerdo con datos del INEGI (2010), en este municipio había 4800 operadores de máquinas industriales de costura. Se trata de un mercado de trabajo relativamente pequeño comparado, por ejemplo, con TorreónGómez Palacio, donde hay cerca de 8 mil que desempeñan dicha ocupación, o en el municipio de Tehuacán, Puebla, donde son más de 10 mil. Otra diferencia importante es que en Torreón-Gómez Palacio hay más empresas grandes, mientras que en Tehuacán predominan las pequeñas y micro, debido a que la subcontratación es una práctica muy extendida, sobre todo, en los procesos más intensivos en mano de obra, como es el deshilado ${ }^{14}$ (López et al. 2011).

\footnotetext{
${ }^{14}$ También, se puede señalar que en los municipios de Torreón-Gómez Palacio y en el de Tehuacán predomina la fabricación de pantalones, y en Tijuana la de camisetas y uniformes.
} 
En la observación en campo fue posible identificar talleres a domicilio, subcontratados por las compañías instaladas en Tijuana, o que tienen un proveedor directo en California, Estados Unidos. Y en ellos laboran principalmente mujeres; es común el trabajo familiar sin pago y a destajo o temporal. La ubicación de los talleres hace difícil su identificación, pues están en colonias poco consolidadas y algunas marginadas. En cambio, las empresas del directorio se encuentran en parques industriales (Pacífico, Las Brisas y Los Pinos, entre otros), se concentran en un área muy consolidada y bien comunicada de la ciudad, a lo largo de la avenida Gustavo Díaz Ordaz, una de las más antiguas e importantes de la ciudad, y las más grandes tienen una cartera de talleres a los que subcontratan, ya sea para completar un volumen de mercancía o en función del tipo de prenda, por lo regular las más difíciles de confeccionar. ${ }^{15}$

La mayoría de las empresas se dedica al ensamble de ropa, que distribuye en tiendas departamentales en el condado de San Diego ${ }^{16}$ (Jc Penny, Walmart, Macy's y Sears, etcétera). Algunas producen ropa de marca que se vende en boutiques de Estados Unidos, y otras extienden su mercado hacia países de Europa, por ejemplo la estadounidense ${ }_{\text {STj }}{ }^{17}$ es una de las que integra en su planta mayor complejidad del proceso productivo, está ubicada en el Parque Industrial Pacífico. En STj se fabrica ropa exclusiva de diseñador, parte de la materia prima (telas) se produce ahí mismo, y tiene una división en donde se confeccionan accesorios con uso intensivo de mano de obra, sobre todo femenina. Por lo general, las cadenas de producción a las que pertenecen las empresas en Tijuana tienen un alcance regional, sólo una de las grandes es eminentemente mundial. La región de California constituye su mercado principal, por lo que su dinámica está muy vinculada al comportamiento de los consumidores estadounidenses.

En cuanto a las relaciones laborales, sobre todo se establecen a través de sindicatos blancos, por lo que pocas veces los trabajadores tienen relación directa o conocen al líder sindical, en otros casos

${ }^{15}$ Entrevista con Efraín (marzo de 2011).

${ }^{16}$ Incluso algunos talleres a domicilio reciben pedidos directos de contratistas de estas tiendas, que llegan a Tijuana a buscar proveedores.

${ }^{17}$ El nombre se omitió por motivos de confidencialidad, en adelante sólo se utilizan siglas para identificar a las empresas. 
se trata de contratos individuales, esto depende del tamaño de las empresas. En las pequeñas y micro las relaciones laborales tienen un grado mayor de individualización y escasa regulación (en las entrevistas, por ejemplo, se sabía poco de las prestaciones y del tipo de contrato). En los talleres a domicilio, las personas trabajan a destajo y en ocasiones pueden hacerlo sin pago. ${ }^{18}$

Otras diferencias relativas a las condiciones laborales se refieren a las prestaciones: en las firmas grandes, además de las establecidas por la Ley Federal del Trabajo, se ofrecen servicios adicionales. Por ejemplo, en sTj se les ha brindado transporte, comedor y guardería, lo que no se encuentra en otras de menor tamaño.

El ritmo de la actividad productiva de las empresas en Tijuana está determinado por la demanda de ropa en California, por lo que la crisis económica reciente en Estados Unidos ha golpeado de forma significativa a esta industria. Los principales efectos han sido en la disminución de los empleos, la caída de los salarios y la pérdida de prestaciones. El cierre de maquiladoras del vestido y textiles en los municipios fronterizos fue muy importante, mientras que en el año 2000 se reportaban 306 establecimientos, en 2006 eran 160 y en 2010 hubo un ligero repunte al incrementarse a $197^{19}$ (INEGI 2011 ). En las entrevistas se reportó la desaparición de líneas de producción y el despido de trabajadores: en 2008 la empresa tiu liquidó a cerca de 150, la stj, que hace diez años empleaba a 1200 personas, hoy en día tiene alrededor de 700 .

En lo que respecta a los salarios, una de las formas de pago de las empresas es a destajo, por ejemplo tIU paga un sueldo base de 500 pesos semanales, a lo que se suma en épocas de auge el pago a destajo, que depende de la productividad diaria del trabajador. En otras el pago es sólo a destajo y no hay un salario base. En este sector todas las tareas tienen un precio establecido, que puede variar a

18 Como en el caso de Yolanda, quien recién llegó a Tijuana de su tierra natal, empezó a laborar en un taller de confección de una vecina, quien ayudó a su esposo a encontrar empleo y sólo le daba a cambio comida o una despensa, durante más de dos años no recibió pago.

${ }^{19}$ Los operadores de máquinas industriales de costura aumentaron en el mismo periodo, de 3855 a 4 807; algunas hipótesis al respecto se relacionan con la dinámica de la actividad productiva en la región económica Tijuana-San Diego-Los Ángeles. Es posible que se mantenga cierta ventaja de inversión en Tijuana, mientras que en el lado estadounidense se ha presentado un declive del sector, por lo que algunas fábricas se reubicaron en esta ciudad. 
lo largo del año, por lo que las variaciones en la remuneración de los y las trabajadoras pueden ser significativas. De acuerdo con la información recabada en las entrevistas, en la década de 1990 los y las costureras podían llegar a ganar hasta 1900 pesos semanales, mientras que en los últimos años la cantidad se ha reducido a la tercera parte o menos.

Aunque la base fundamental del trabajo en estas empresas descansa sobre los operarios de las máquinas industriales de costura, hay otros puestos remunerados con un salario fijo, que es menor al de las y los costureros, como el de los tendedores, planchadores, deshiladores y empacadores. Los cortadores también lo reciben pero, por lo general, es mayor al de los mencionados antes; dichos puestos se han tornado cada vez más escasos, de acuerdo con información en las entrevistas, ya que las empresas han preferido dedicarse al ensamble.

El pago a destajo le confiere a los y las trabajadoras un sentido de autonomía muy particular, y contribuye al proceso de individualización de las relaciones laborales, puesto que se envuelven en dinámicas de autoexploración. Según algunos de los testimonios, lo anterior se expresa en su percepción de que en la línea de producción trabajan sin supervisores. Sin embargo, otros relatos dan cuenta de la vigilancia cotidiana tanto de los ritmos de trabajo como de la calidad de las tareas, por parte de supervisores y gerentes.

La heterogeneidad laboral en la industria de la confección tijuanense, entendida como multiplicidad de tipos de relaciones sociales en el trabajo, pareciera reproducirse en este contexto en el que las empresas combinan prácticas de flexibilización interna (variaciones en el número de empleos, en el salario y en la jornada) y externa (subcontratación). La persistencia de talleres ${ }^{20}$ es el resultado de dicha flexibilidad laboral en Tijuana y en el otro lado de la frontera.

Según el INEGi (2010) los operadores de máquinas industriales de costura contaban con prestaciones como: vacaciones (86 por cien-

\footnotetext{
${ }^{20}$ En el trabajo de campo se identificaron dos casos de costureras que trabajaban en su taller, con poca relación con las fábricas, y confeccionaban prendas de vestir para el mercado local o regional. Una de ellas se dedicaba a coser vestidos de gala y sus principales clientes venían de San Diego o de Los Ángeles. La otra distribuía sus prendas en el mercado sobre ruedas de su colonia.
} 
to), aguinaldo (91), reparto de utilidades (87) y servicios médicos (88). Si bien, estos datos revelan un grado importante de formalización en esta ocupación dentro de la fabricación de prendas de vestir, la información sobre las horas laborales muestra la importancia tanto de la informalidad, por las 1194 personas que trabajan menos de 48 horas semanales (25 por ciento), como de la sobrecarga de trabajo de las 1385 que lo hacen por más de 48 horas a la semana (29 por ciento).

\section{La diversidad laboral: migración, género y generación}

La preocupación por la creciente incorporación de las mujeres a los mercados de trabajo, junto con la precarización del empleo, ha generado un intenso debate acerca del significado de esta feminización. Por tanto, se considera que la incorporación femenina al trabajo remunerado ha constituido una tendencia de abaratamiento de la fuerza laboral en México y en las maquiladoras. Sobre esta discusión sólo interesa destacar que la presencia femenina en la PEA y en la industria maquiladora ha obligado a replantear el modelo ideal de trabajo de la época de sustitución de importaciones: los trabajadores eran hombres jefes de hogar y únicos proveedores. En particular, ha llevado a retomar el tema de la diversidad y las diferencias en la orientación y el significado del trabajo, así como la relación entre los ámbitos de producción y reproducción.

La diversidad laboral en este mercado de trabajo se define por las diferencias entre la población trabajadora, pero se refiere más a la distancia que existe hoy en día respecto al modelo tradicional de trabajador. El análisis sobre dicha diversidad empieza a ser importante con la creciente incorporación de la mujer al trabajo asalariado, ocurrida durante la segunda mitad del siglo pasado, ${ }^{21}$ y el género ha sido una de sus principales raíces, pero en el caso de la costura ha ocurrido un proceso inverso, pues se había considerado

${ }^{21}$ En Guadarrama y De la O (2006) se concluye, a la luz de la revisión de las investigaciones sobre género y trabajo en América Latina, la importancia de estudiar los procesos de trabajo considerando la noción de sujetos más complejos y sexuados. 
históricamente como un oficio femenino. Sin embargo, los procesos de industrialización, sobre todo en la etapa reciente, en la que predomina el ensamble de piezas, ha habido una masculinización del empleo. Para 1985, en los municipios fronterizos en este ramo había tres hombres por cada diez mujeres, en 2003 la relación aumentó más del doble: siete por cada diez.

La masculinización se encuentra asociada a factores internos y externos a la fábrica, entre los primeros están los cambios en los procesos productivos y las condiciones de trabajo; la intensificación de las jornadas favorece la entrada de hombres. También se observa una división sexual del trabajo, de acuerdo con ciertos requerimientos físicos, pues algunos puestos son ocupados predominantemente por hombres o por mujeres. Por ejemplo, para el corte de las telas se prefiere a los hombres, en las áreas donde se confeccionan grandes piezas como manteles; mientras que se elige a las mujeres para las tareas más minuciosas como la confección de accesorios de prendas de vestir. ${ }^{22}$

Otra raíz de la diversidad en este mercado de trabajo es la migración, que es parte definitoria de las experiencias vitales entre las personas que participan en estos empleos. De acuerdo con los datos del INEGI (2010), en el municipio de Tijuana 90 por ciento de operarios, los de máquinas de costura, bordado y de corte para la elaboración de prendas de vestir, nacieron en otro estado de la república; ${ }^{23}$ predominan los del Distrito Federal, Sinaloa, Michoacán, Chiapas, Sonora y Jalisco. ${ }^{24}$ En estos casos, el trabajo forma parte de un proyecto de búsqueda de mejores condiciones de vida, a través de la movilidad geográfica. En tales procesos la movilidad social puede ser ascendente o descendente. Según el orden de racionalidad desde el que se sitúe cada persona, de tal manera que los y las trabajadoras recorren circuitos sinuosos y complejos en su vida económica, por

\footnotetext{
${ }^{22}$ Los y las trabajadoras entrevistadas explicaban que la preferencia de hombres o mujeres en los puestos de costura por parte de la gerencia se debía a que se valora la responsabilidad como un atributo femenino, y a la resistencia física como uno masculino.

${ }^{23}$ Esta característica es poco común en los municipios de Torreón y Gómez Palacio, donde 10 por ciento de operarios(as) de máquinas de costura, bordado y 20 de corte para la elaboración de prendas de vestir nacieron fuera del estado.

${ }^{24}$ En la muestra intencional de la investigación empírica, que incluyó 27 casos, sólo 4 fueron originarios de Tijuana.
} 
lo que en parte con frecuencia se encuentran trayectorias laborales itinerantes.

La precariedad que se observa en el terreno objetivo no corresponde con la vivida entre los y las trabajadoras, en este nivel de análisis se observan narrativas muy contrastantes, en las que las diferencias en términos de recursos sociales y culturales ${ }^{25}$ significan distintas posibilidades de acceder a oportunidades de mejoramiento relativo de las condiciones de vida y de empoderamiento (en las mujeres).

\section{Figura 2}

Municipio de Tijuana: principales características sociodemográficas de los operadores de máquinas industriales de costura

\begin{tabular}{|c|c|c|c|c|c|c|}
\hline & \multicolumn{2}{|c|}{ Edad } & \multicolumn{2}{|l|}{ Sexo } & \multicolumn{2}{|c|}{ Lugar de nacimiento } \\
\hline & $12-30$ & 31 y más & Mujeres & Hombres & $\begin{array}{c}\text { En el } \\
\text { estado }\end{array}$ & $\begin{array}{c}\text { Fuera del } \\
\text { estado }\end{array}$ \\
\hline Total & 1556 & 3251 & 3320 & 1487 & 436 & 4371 \\
\hline \multirow[t]{3}{*}{$\%$} & 32 & 68 & 69 & 32 & 9 & 91 \\
\hline & \multicolumn{5}{|c|}{ Estado civil } & \\
\hline & $\begin{array}{l}\text { Unión } \\
\text { libre }\end{array}$ & $\begin{array}{c}\text { Casados } \\
\text { (as) }\end{array}$ & $\begin{array}{l}\text { Separados(as)/ } \\
\text { divorciados(as) }\end{array}$ & $\begin{array}{l}\text { Viudos } \\
\text { (as) }\end{array}$ & $\begin{array}{c}\text { Solteros } \\
\text { (as) }\end{array}$ & \\
\hline Total & 1373 & 1783 & 792 & 71 & 788 & \\
\hline$\%$ & 29 & 37 & 16 & 32 & 16 & \\
\hline \multicolumn{4}{|c|}{ Escolaridad } & & & \\
\hline & $\begin{array}{l}\text { Primaria } \\
\text { o menos }\end{array}$ & Secundaria & $\begin{array}{c}\text { Más de } \\
\text { secundaria }\end{array}$ & & & \\
\hline Total & 529 & 2435 & 1788 & & & \\
\hline$\%$ & 11 & 51 & 32 & & & \\
\hline
\end{tabular}

Fuente: elaboración propia, a partir de los datos del INEGI (2010).

${ }^{25}$ Es importante recordar que la costura pasó de ser un oficio a la fragmentación de las tareas y a la automatización de las máquinas de coser, de tal modo que un puesto en la línea de producción se especializa en la costura de una parte de la pieza de vestir. Los requerimientos de calificación de las tareas son limitados, por lo que el nivel escolar entre los trabajadores es bajo. En Tijuana, 50 por ciento de la población ocupada en la confección de prendas de vestir tiene estudios de primaria o no tiene instrucción alguna, cerca de la tercera parte tiene secundaria y menos de una cuarta parte preparatoria o bachillerato INEGI (2000). 
Una tercera fuente de diversidad es la edad de las personas empleadas en estas fábricas, las diferencias están relacionadas con el curso de vida, el cual entre los jóvenes corresponde a etapas de formación de la familia o a la permanencia en la paterna o materna. Sin embargo, en este estudio es importante distinguir entre jóvenes y adultos, por la coyuntura en la que se insertan en el mercado de trabajo. En efecto, los jóvenes tienen trayectorias laborales más cortas, pero también ingresaron al trabajo en una época de crisis, de finales de los años noventa a la fecha. En cambio, los adultos empezaron con el auge de las maquiladoras en Tijuana. ${ }^{26}$

De acuerdo con la figura 2, uno de los perfiles sociodemográficos más representativos de los operadores de máquinas industriales de costura es ser mujer (69 por ciento), migrante (91), mayor de 30 años (68), casada o en unión libre (53) y con escolaridad de secundaria (51).

\section{La precariedad vivida: diferencias de género y generación en un contexto fronterizo}

En este apartado se presenta una descripción de las experiencias de vida de algunas de las personas entrevistadas durante 2010 y 2011. Los casos se seleccionaron a partir de una lectura de cada narrativa, y se analizó el contenido para identificar los temas centrales de su experiencia, de tal manera que un elemento distintivo fue la migración individual o familiar y cómo cada uno asume esta vivencia. Por ello, se decidió hacer una interpretación del sentido del trabajo tomando como eje de análisis principal la migración y las diferencias de género y generación. En una segunda lectura se reconstruyeron los perfiles de algunos casos paradigmáticos, ${ }^{27}$ los cuales se distinguen por representar experiencias compartidas con otros o que muestran situaciones de contraste. Es importante aclarar que la estructura de este apartado responde a un ejercicio inductivo, es decir, se partió

\footnotetext{
${ }^{26}$ Según el INEGI (2010), se registraron 1556 operadores(as) de máquinas industriales de coser menores de 30 años, mientras que la mayor concentración, 3251 , fue entre los adultos (mayores de 30).

${ }^{27}$ Son ejemplos que funcionan como referentes para mostrar diferentes sentidos del trabajo y formas de vivir la precariedad. Todos los nombres son seudónimos.
} 
de los casos y la interpretación de las entrevistas para presentarlos de esta manera.

Los jóvenes acompañantes o segunda generación de migrantes

Más que un rango de edad, interesa describir a estos jóvenes costureros en relación con sus antecedentes migratorios y las formas de otorgarle significado a su inserción laboral en las fábricas de confección. Los que llegaron a Tijuana, como hijos de familia o que nacieron en la ciudad, pero de padres inmigrantes, se enfrentan a la necesidad de trabajar desde una posición distinta a la de sus padres. Entre ellos, es común que su trayectoria laboral empiece como parte de una estrategia para completar los ingresos de la familia, porque son los padres o madres quienes los recomiendan en el lugar de trabajo, así como también les tramitan un permiso ante el municipio, para que los menores de edad laboren bajo su tutela en la misma fábrica. Para los jóvenes, el empleo en las fábricas forma parte de su entorno, en este sentido se han constituido inercias en el lugar, que facilitan la entrada a estos mercados de trabajo. Y en su imaginario, ser costureros es una etapa de transición, en el futuro ellos se ven en otra parte, porque están estudiando, tienen como proyecto cruzar la frontera o debido a que su visión de futuro se encuentra indefinida. En estos casos, la precariedad es percibida como parte de un contexto heredado, pero es una condición que pueden superar en su transición hacia la adultez, aunque con poca claridad del camino a seguir; ${ }^{28}$ además, las diferencias entre las mujeres y los hombres se hicieron poco visibles.

La migración y las formas de inserción laboral masculina

Distintos estudios han documentado los vínculos entre la migración interna y la internacional; ${ }^{29}$ en las entrevistas realizadas, Tijuana es vista como un destino transitorio, como una ciudad de paso en el

\footnotetext{
${ }^{28}$ En este perfil se menciona las narrativas de jóvenes entrevistados en esta circunstancia de vida, pero debido a su corta experiencia laboral se omitieron mayores referencias.

${ }^{29}$ En Lozano (2003) se plantea esta relación también, pero es difícil presentar conclusiones al respecto en este trabajo, para ello se requiere otro tipo de estudio que se base en datos de una muestra representativa.
} 
camino a Estados Unidos, pero que las oportunidades brindadas por las maquiladoras, sobre todo durante la década de 1990, permitieron a los hombres quedarse ahí; algunos ingresaron directamente a dichas plantas y otros a empleos precarios de fácil acceso, como la construcción. Una característica encontrada en los antecedentes de las personas entrevistadas fue el origen rural de sus familias, entre los hombres se observó el cambio de perfil de agricultores a obreros de maquila, ${ }^{30}$ ya sea una transición directa o con algunas experiencias previas en otros empleos y sectores.

Entre los hombres se identificaron dos referentes de este perfil: uno exitoso y otro de fracaso y exclusión. Sin embargo, se pueden considerar como los dos polos de un continuo en el que, según los recursos disponibles, los trabajadores pueden encontrar formas de enfrentar las condiciones precarias del mercado de trabajo. También es importante destacar que los dos casos seleccionados ilustran la existencia de una estrategia de empleo de las empresas, en la que hay un núcleo base de trabajadores que pueden hacer carrera dentro de ella y otro conjunto que tiene una relación laboral más flexible o temporal; tal como ya lo habían señalado Doeringer y Piore (1971) en su planteamiento sobre la existencia de un mercado interno y uno externo. Por otra parte, un elemento que puede influir en la configuración de trayectorias laborales de empresa es el momento de la inserción, pues en época de auge habrá más oportunidades de permanecer en el empleo y cuando haya crisis éstas tenderán a disminuir. Asimismo, el tamaño de las empresas puede tener un papel importante ya que, como se señaló, éstas ofrecen mejores condiciones, en el caso del trabajador que hizo una carrera dentro de alguna fue en una de las más grandes empresas de Tijuana.

Perfil 1: Ismael, hombre con experiencia de migración rural-urbana ${ }^{31}$ y movilidad

Ismael se inició como trabajador familiar en la agricultura a los ocho años, pero sin pago; su trayectoria laboral es típica de los procesos

\footnotetext{
${ }^{30}$ También se observó que hay un peso importante de la socialización primaria en el campo, entre los entrevistados existía la constante de que sus padres habían sido trabajadores agrícolas.

${ }^{31}$ Es importante aclarar que en otros estudios se ha dado cuenta de migración urbanaurbana, en la que hubo hombres que tenían otro oficio y que en su lugar de origen su empleo
} 
de migración campo-ciudad, pasó de agricultor a operador de una máquina de coser en la fábrica tiu, donde labora desde hace 15 años; en 1995, cuando llegó de Oaxaca. Además, se trata de una trayectoria de carrera, hoy es supervisor de línea y uno de los que reportaron mayores ingresos, su perfil responde al de jefe proveedor de familia con movilidad socioeconómica, pues sus hijos cuentan con mayor escolaridad que él.

Junto con este caso, que forma parte de un núcleo reducido de trabajadores con estabilidad laboral y que pudieron acceder a rutas de movilidad interna en la empresa, se observan otras experiencias de hombres migrantes que afrontan condiciones más difíciles. Por ejemplo, se eligió la narrativa de Efraín, porque se trata de un perfil extremo en relación con Ismael. A través de su experiencia es posible identificar las situaciones de riesgo y acumulación de desventajas a las que algunos se enfrentan.

Perfil 2: Efraín, hombre con experiencia de migración rural-urbana y exclusión

Efraín nació en una localidad rural del estado de Puebla, antes de emigrar rumbo a Estados Unidos había trabajado en la Ciudad de México como mesero; sólo estudió la primaria y empezó su vida en pareja muy joven, tuvo su primer hijo a los 17 años. En su lugar de origen trabajó por primera vez en una fábrica de ropa, donde aprendió el oficio de cortador. Llegó a Tijuana a principios de los años noventa, con la idea de cruzar la frontera, pero su contacto lo dejó en la ciudad sin recursos, por lo que empezó a trabajar como ayudante de albañil. Después encontró empleo como cortador en una fábrica de costura, cuando logró cierta estabilidad y con la ayuda de su patrón regresó a Puebla a traer a su esposa y cuatro hijos. Desempeñó el mismo puesto en tres o cuatro fábricas distintas, y se salió de la primera porque por medio de otros cortadores se dio cuenta que le pagaban mucho menos que a ellos en otras maquiladoras; durante años recibió un sueldo muy inferior al que se ofrecía en otras fábricas. A principios del año 2000 se lastimó la espalda du-

se fue precarizando por lo que decidieron buscar otras oportunidades de desarrollo en el norte de México. 
rante la jornada, pero no acudió al seguro social, por desconfianza y por consejo del patrón quien le sugirió atenderse con un sobador. Este accidente le provocó un deterioro muy grave de la salud, que devino en una operación de columna que lo puso en riesgo de ya no caminar. Cuando regresó a la fábrica fue objeto de discriminación por su incapacidad para adaptarse al ritmo de trabajo, lo quisieron cambiar a un puesto de menor remuneración y estatus. ${ }^{32}$ Finalmente renunció, y enfrenta una situación de vulnerabilidad extrema por su condición de salud, que ya le impide mantener a su familia, por los gastos que conlleva, así como debido a su alcoholismo; esto obligó a la esposa e hijos a regresar a Puebla en fecha reciente. También perdió la vivienda que había construido en un terreno adjudicado hacía años por un proceso de invasión. En la actualidad consiguió un empleo en el área de corte de una maquila, porque decidió ocultar su lesión en la entrevista, pero vive con el temor constante de perderlo o sufrir un accidente que empeore su salud.

Algunas de las carencias detectadas en este caso, desde el momento de su llegada a Tijuana, son de naturaleza distinta y tienen que ver con la vulnerabilidad del migrante; a la falta de recursos económicos se suma la inexistencia de redes sociales de apoyo y un desconocimiento del mercado de trabajo. En cambio, en otros estudios se ha descrito el papel de las redes y cómo la migración de personas de una misma comunidad, algunos con lazos familiares, permite la trasmisión de conocimiento acerca de la ciudad y el empleo, por lo que disminuyen las situaciones de abuso y vulneración (Solís 2009).

A través de esta narrativa se pueden entender las formas en que los empleadores violan los derechos laborales de los trabajadores por su condición de inmigrantes; los riesgos físicos y los mecanismos que se utilizan para seleccionar a los más eficientes, en los que se involucran los mismos compañeros; así como la desconfianza hacia las instituciones públicas de salud. En suma, se observó la ausencia de la acción del gobierno y del Estado para garantizar los derechos

\footnotetext{
32 El estatus de los puestos depende del grado de dificultad y la importancia en el proceso productivo, las ocupaciones de tendedor y deshilador tienen menos prestigio y emplean a personas menos calificadas, entre ellos adultos mayores, como Laura, quien entró a laborar a los 55 años, para ayudar a su hijas a incrementar los ingresos de su hogar, para hacerle frente a los problemas recientes de encarecimiento de la vida.
} 
laborales de este sector de población, así como un problema de exigibilidad de los derechos laborales y sociales.

Otra dimensión de la vulnerabilidad de este caso es la relacionada con el alcoholismo, como una enfermedad que trastoca toda la existencia y que pudiera estar vinculada con los mandatos de la masculinidad hegemónica. Este conjunto de dificultades van formando un circuito de acumulación de carencias que llevan a las personas a la exclusión social.

La migración y las formas de inserción laboral femenina

En el análisis de la experiencia femenina de trabajo en la costura, las características del proceso migratorio son determinantes, por ello se hizo la distinción entre las mujeres que decidieron emigrar en busca de trabajo y quienes se trasladaron en pos de la reunificación familiar, es decir, por seguir al esposo en su camino hacia el norte. En el primer perfil se observa una inserción laboral fundada en un proyecto más personal de construcción de autonomía económica. El segundo puede tener dos vertientes, por un lado, las mujeres que trabajan como una estrategia familiar y cuyo ingreso es complementario al principal de sus esposos, por lo tanto, su papel como proveedoras es secundario, y el empleo se ajusta a sus responsabilidades en el ámbito de la reproducción, que sigue siendo la centralidad de su vida cotidiana. Para otro conjunto de mujeres, la inserción laboral ocurrió como parte de un punto de inflexión en su curso de vida familiar, pues si bien llegaron a Tijuana siguiendo al esposo, las circunstancias las llevaron a la separación o al divorcio, por lo que le otorgan sentido a su empleo en la fábrica a partir de la necesidad de reconstruir su vida y a la de asumirse como jefas de hogar.

Perfil 3: Karla, mujer con experiencia de migración laboral

Karla llegó a Tijuana desde Honduras a mediados de los años noventa; su objetivo era cruzar a Estados Unidos, pero tuvo la oportunidad de empezar a trabajar, y ha permanecido como costurera 17 años, en siete fábricas distintas. El motivo para emigrar fue la necesidad de enviarles recursos a su hijo y sobrinos, que se quedaban con sus padres en su ciudad natal. Ella era madre soltera y casarse 
estaba fuera de sus expectativas, pues tenía como referente negativo las experiencias de violencia e irresponsabilidad de las parejas de sus hermanas. Sin embrago, en su camino al norte entabló una relación de pareja, ambos llegaron a Tijuana y parte de su decisión para quedarse fue el embarazo. En los 17 años que tienen en esta ciudad lograron comprar una casa y mantener en la escuela a sus dos hijos. Sin embargo, la crisis de los últimos años ha modificado sus condiciones de vida y los ha expuesto a una situación de vulnerabilidad, pues su esposo perdió el empleo recientemente y sus ingresos se redujeron en forma drástica. Ella estuvo desempleada cerca de dos años y su salario a destajo en la costura apenas le permite ganar entre 150 y 200 pesos diarios. Perdieron la casa, que obtuvieron por medio del Instituto del Fondo Nacional de la Vivienda para los Trabajadores, y tienen que pagar en la que ahora habitan.

El caso de Karla se ajusta al perfil de migración laboral femenina, para ella es central su experiencia de tránsito desde Honduras hasta Tijuana, y las motivaciones para dejar su país en busca de una mejora económica y de un proyecto de autonomía personal, debido a sus referentes de matrimonios fracasados por abandono o violencia doméstica. En esta narrativa, la reconstrucción identitaria de género juega un papel central, y permite pensar la experiencia laboral de manera distinta, pues para las mujeres representa un recurso fundamental para su empoderamiento. Al mismo tiempo, se encontró la contradicción de los empleos precarios en los que el desarrollo femenino encuentra un límite, sobre todo en periodos de crisis, tal como el que ha atravesado el sector de la confección en los últimos años en la frontera norte de México.

Perfil 4: Sandra y Yolanda, mujeres con experiencia de migración familiar

Sandra y Yolanda llegaron a Tijuana cuando seguían a sus parejas que iban a Estados Unidos, las dos son del mismo poblado de Nayarit, donde sus maridos eran jornaleros y ahora son albañiles. Las dos viven en la misma ladera de uno de los cerros del este de la ciudad, en una colonia urbanizada por el último movimiento urbano popular en Tijuana; ellas obtuvieron su solar mediante la invasión, años después de la primera gran toma de terrenos. Sus casas se encuentran en un espacio que pareciera estar fuera de la ciudad, mantienen 
relaciones de reciprocidad entre los vecinos, muchos son parientes o paisanos. Ellas trabajan en la costura, y aunque sus salarios pueden ser mayores que los de sus maridos, éstos siguen siendo la cabeza de familia. Aunque Yolanda ha sobrevivido con su trabajo en las fábricas y luego con las labores de costura que hace en casa, con una máquina que le compró su esposo, tiene la posibilidad de retornar a su pueblo, en buena medida debido a las dificultades económicas que han vivido en los últimos años.

Este perfil se encontró entre mujeres que llegaron a Tijuana como esposas, pero que forman parte de familias con dos ingresos. Ellas mantienen la centralidad del rol tradicional de madres y responsables del trabajo doméstico, porque sus ingresos se conciben como complementarios y de los que se puede prescindir, y continuamente ajustan su actividad laboral remunerada a las necesidades de crianza de los hijos.

Perfil 5: Jacinta, mujer con experiencia de migración familiar y ruptura

El caso de Jacinta es muy distinto, ella también siguió a su marido, pero ha transitado por un proceso de reconstrucción identitaria y de empoderamiento. Vivió un matrimonio forzado, pues fue robada por un hombre mucho mayor que ella, en Guerrero, y sus propios padres la obligaron a casarse. Después de tener cinco hijos y de establecerse en Tijuana, su esposo la dejó por otra mujer. Aunque había trabajado en algunas maquiladoras cuando recién llegó a Tijuana, tras la separación se integró de nuevo al trabajo remunerado en 2004. A sus 45 años ingresó a una fábrica de costura, y después de cuatro años obtuvo un crédito para vivienda. Al poco tiempo fue liquidada, y con la indemnización compró dos máquinas de coser. Dos años más tarde intentó volver a trabajar en la fábrica, pero el salario era tan bajo que prefirió montar su propio taller, donde actualmente confecciona prendas de vestir que una persona le distribuye en el tianguis de su colonia. Vive sola y sus hijos ya son mayores de edad, por lo que tiene un fuerte sentido de autonomía, que vive como un proceso de liberación.

La circunstancia en la que se empleó Jacinta y su ruptura, respecto a su vida como madre y esposa, contribuyen a entender por qué el trabajo significa un medio imprescindible para lograr su au- 
tonomía económica. Sin embargo, la precarización del empleo en la confección ha implicado inestabilidad y escasez, para enfrentar esta situación Jacinta optó por trabajar por cuenta propia y sumarse a la informalidad. Por otra parte, representa una ventaja estar sola, pues recibe apoyos de sus hijos ya mayores, entonces sus necesidades económicas son menos apremiantes que las de mujeres más jóvenes y en otra etapa de su vida.

Aquí, en el análisis de los casos se consideró la orientación hacia el trabajo en relación con la biografía sociolaboral, se destacaron los perfiles con situaciones contrastantes a fin de dar cuenta de los procesos vividos individualmente, y que llevan a distintos mecanismos o formas de encarar las condiciones de precariedad que encuentran en estos empleos. Hubo trayectorias sociolaborales de movilidad o de exclusión en los hombres entrevistados; procesos de autonomía y empoderamiento de las mujeres versus mecanismos de reproducción de la condición del trabajo femenino como secundario. En los jóvenes, las diferencias de género pasan a un segundo plano para destacarse como un momento de transición, de incertidumbre pero en el que se gesta un cambio generacional respecto a los padres en Tijuana, cuya experiencia de vida por lo común ha sido en el campo. Es importante aclarar que si bien este es un estudio de caso, de carácter exploratorio, permite identificar formas de enfrentar la precariedad desde las distintas circunstancias de vida y con recursos acumulados a lo largo de ella. Aunque se destacaron perfiles contrastantes, hay que insistir en que entre un polo y otro hay grados y combinaciones, que ilustran desde los sujetos las formas de reproducción de un mercado de trabajo precario, así como la generación de estrategias alternativas para sobrellevar la precarización, como la configuración de proyectos de retorno y el trabajo por cuenta propia.

\section{Conclusiones}

El objetivo de este artículo fue analizar los procesos de precarización laboral en un mercado de trabajo local y desde una perspectiva sociocultural, lo cual implicó considerar las condiciones del con- 
texto y la experiencia de vida de los sujetos que participan en su construcción.

Los procesos que han dado lugar a la precarización laboral se relacionan con la reproducción o extensión de la heterogeneidad estructural y con los de proletarización de amplios sectores de población de las zonas rurales del país. Las prácticas de flexibilización productiva, tanto interna como externa a las empresas, repercuten en la precarización del mercado de trabajo. En la confección de prendas de vestir, la subcontratación y el pago a destajo han sido formas de ajustar la actividad productiva de las fábricas a los cambios en la demanda, que se configura principalmente desde la región transfronteriza de San Diego. El papel limitado que juega Tijuana en la producción global de prendas de vestir ha llevado al predominio de empresas pequeñas y medianas, que se encuentran menos reguladas que las grandes.

La importancia de la condición migratoria de las personas que se insertan en estos mercados de trabajo es un aspecto fundamental para entender el sentido que le otorgan al trabajo y los recursos sociales y culturales con los que enfrentan la precariedad. Estas circunstancias explican en el plano microsocial su persistencia y la vulnerabilidad acentuada en la última década, pues la otrora ciudad de oportunidades de los años noventa tiende a desaparecer, para dar lugar a una de riesgo, pobreza y violencia social.

En el plano microsocial se observaron distintas capacidades y formas de negociar la precariedad. La inserción de algunos jóvenes con relaciones de parentesco entre el personal de las fábricas de confección, y la trasmisión generacional del oficio de la costura da cuenta de ciertos mecanismos de continuidad en este mercado de trabajo. Algunas mujeres reproducen prácticas que podrían estar manteniendo intactos los límites de estos empleos, como alternativas de desarrollo personal y autonomía económica, pues asumen su participación secundaria en la manutención del hogar, por lo que muchas veces el trabajo juega un papel coyuntural y poco importante en su vida cotidiana; mientras que para otras ha sido un medio para superar su condición femenina de desventaja, pero que les impone límites a su posible movilidad social e incluso pone en riesgo su bienestar. 
La emigración campo-ciudad, como parte del proceso de proletarización en México, marca la trayectoria laboral masculina y relativiza la precariedad, ya que las expectativas de vida rural se tornan insostenibles debido al abandono del campo, por décadas de políticas neoliberales de ajuste y de crisis económicas reiteradas. Además, la inserción masculina en estos mercados de trabajo pareciera reforzar el imaginario de la frontera como un espacio de paso, pues estos empleos constituyen un refugio -muchas veces temporal- frente a la falta de otras opciones en su ciudad de origen, o por las mayores dificultades para el cruce de la frontera.

La limitación del espacio en este texto hizo imposible un análisis detallado de algunas evidencias de prácticas de resistencia frente a la precarización laboral. Aunque es pertinente señalar que las respuestas entre los y las trabajadoras están poco articuladas y son coyunturales; mientras que los proyectos colectivos de más alcance son muy puntuales, pues la acción sindical es escasa y sólo algunas organizaciones de la sociedad civil mantienen espacios de defensa de los derechos laborales de esta población, como el Colectivo Ollin Calli, el Colectivo Chilpancingo Pro Justicia Ambiental, la Casa de la Cultura Obrera y el Centro de Información para Trabajadores y Trabajadoras.

Recibido en septiembre 2012 Aceptado en mayo de 2013

\section{Bibliografía}

Avenel, C., y F. Thibault. 2006. Précarités et insécurité sociale, Problémes politiques et sociaux 921. París: La Documentation Francaise.

Bair, Jennifer y Gary Gereffi. 2001. Local Clusters in Global Chains: The Causes and Consequences of Export Dynamism in Torreon's Blue Jeans Industry. World Development 29 (11): 1885-1903.

CNIV. 2011. Memoria estadística 2009. La industria del vestido. México. 
Castel, Robert. 1997. Las metamorfosis de la cuestión social. Una crónica del asalariado. Buenos Aires: Paidós.

De la O, María Eugenia. 2006. El trabajo de las mujeres en la industria maquiladora de México: balance de cuatro décadas de estudio. Revista de Antropología Iberoamericana 1 (3): 404-427.

Doeringer, P., y M. Piore. 1971. Internal Labour Markets and Manpower Analysis. Nueva York-Londres: M. E. Sharpe Inc.

García, Beatriz. 2004. Panorama crítico para la industria textil y del vestido mexicana. El Cotidiano 20 (127): 73-84.

García, Brígida. 2009. Los mercados de trabajo urbanos de México a principios del siglo xxi. Revista Mexicana de Sociología 71 (1): 05-46.

Guadarrama, Rocío y María Eugenia De la O. 2006. Género, proceso de trabajo y flexibilidad laboral en América Latina. En Teorías sociales y estudios del trabajo: nuevos enfoques, coordinado por Enrique De la Garza, 289-308. México: Anthropos/Universidad Autónoma Metropolitana.

, Alfredo Hualde y Silvia López. 2010. Precariedad laboral, heterogeneidad ocupacional y desigualdades de género en México en el contexto de crisis actual: una propuesta metodológica. Ponencia presentada en el Xvir Congreso de sociología de la Asociación Internacional de Sociología, Suecia.

Guillén, Arturo. 2005. Integración, heterogeneidad estructural y empleo: el caso de México. Comité para la Anulación de la Deuda en el Tercer Mundo. http:// www. cadtm.org/Integración-heterogeneidad (8 de mayo de 2011).

INEGI. 2000 y 2010. Censo de población y vivienda (microdatos). México. 
2009 y 2011 . Encuesta nacional de ocupación y empleo. México.

2011. Directorio estadístico nacional de unidades económicas. México.

López, Silvia, Marlene Solís y Luis Hernández. 2012. Precariedad y desigualdades de género en la industria de la confección: un estudio de tres regiones. Ponencia presentada en el xxx Congreso internacional de la Asociación de Estudios Latinoamericanos, San Francisco.

2011. Los trabajadores de la industria de la confección en Torreón, Tehuacán y Tijuana. Ponencia presentada en el Seminario Heterogeneidad ocupacional, precariedad laboral y desigualdades de género en México, Ciudad de México.

Lozano, Fernando. 2003. Interrelación entre la migración internacional y la migración interna en México. Órgano Informativo de la Comisión de Derechos Humanos del Estado de México 10 (62): 75-87.

Mora, Minor y Orlandina de Oliveira. 2010. Las desigualdades laborales: evolución, patrones y tendencias. En Desigualdad social. Colección Los grandes problemas de México, coordinado por Fernando Cortés y Orlandina de Oliveira, 101-140. México: Colmex.

Pacheco Gómez Muñoz, María Edith. 2004. Ciudad de México, heterogénea y desigual. Un estudio sobre el mercado de trabajo. México: COLMEX.

Reygadas, Luis. 2002. Producción simbólica y producción material: metáforas y conceptos en torno a la cultura del trabajo. Nueva Antropología XVIII (60): 101-119.

Rojas García, Georgina y Carlos Salas Páez. 2008. La precarización del empleo en México, 1995-2004. Precarización laboral. Revista Latinoamericana de Estudios del Trabajo II época 13(19): 39-78. 
Solís, Marlene. 2009. Trabajar y vivir en la frontera. Identidades laborales en las maquiladoras de Tijuana. México: CoLEF/Miguel Ángel Porrúa.

y Marcela Castañeda. 2013. Jóvenes en la confección: trayectorias laborales y expectativas de vida en la frontera. En Fronteras comparadas: desarrollo, trabajo y migración. México-Estados Unidos/ Marruecos-Unión Europea, coordinado por María del Rosio Barajas y Marlene Solís, 87-115. México: Colef/Juan Pablos Editores. 